DOI $10.35381 / \mathrm{cm} \cdot \mathrm{v} 5 \mathrm{i} 1.262$

\title{
Técnicas de salado en la elaboración de queso artesanal en la zona norte de Manabí
}

\section{Salting techniques in the production of artisan cheese in the northern area of Manabí}

\author{
Rudyard Antonio Arteaga Solorzano \\ r arteaga@utm.edu.ec \\ Universidad técnica de Manabí, Portoviejo \\ Ecuador \\ https://orcid.org/0000-0001-5499-7334 \\ Freddy Alain Mendoza Rivadeneira \\ famendoza@utm.edu.ec \\ Universidad técnica de Manabí, Portoviejo \\ Ecuador \\ https://orcid.org/0000-0003-1457-688X \\ Roy Leonardo Barre Zambrano \\ rolebaz@hotmail.com \\ Universidad Laica Eloy Alfaro de Manabí, Manta \\ Ecuador \\ https://orcid.org/0000-0002-4849-3532 \\ Plinio Abelardo Vargas Zambrano \\ pavargas@utm.edu.ec \\ Universidad técnica de Manabí, Portoviejo \\ Ecuador \\ https://orcid.org/0000-0002-2152-7317
}

Recibido: 15 de agosto de 2019

Aprobado: 15 de septiembre de 2019 


\title{
RESUMEN
}

Se estudiaron los métodos de salado usados en la provincia de Manabí para los cantones de Flavio Alfaro, Chone, Pedernales y el Carmen, se tomaron 245 muestras de la población total de productores de la zona donde se determinó que el $60 \%$ de los productores prefiere el uso de sal en grano gruesa, el $81 \%$ del total de las producciones lecheras realiza el salado después del desuerado, existe una relación directamente proporcional entre el tamaño de la producción y la cantidad de sal utilizada, los Cantones Pedernales, Flavio Alfaro y Chone se caracterizan por una producción de menor a 25 libras por día en el $70 \%$ de las fincas solamente en el Carmen las producciones en el $43 \%$ de los casos son entre 25 y 50 libras diarias, el $17 \%$ de los productores están dispuestos a cambiar de método de salado si este aumenta su rendimiento.

Descriptores: Nutrición animal, ganado vacuno, producto lácteo, industria lechera.

\begin{abstract}
The salting methods used in the province of Manabí for the cantons of Flavio Alfaro, Chone, Pedernales and El Carmen were studied, 245 samples were taken from the total population of producers in the area where it was determined that $60 \%$ of the producers prefer the use of coarse-grained salt, $81 \%$ of the total milk production is salted after desuerado, there is a directly proportional relationship between the size of the production and the amount of salt used, the Pedernales Cantons, Flavio Alfaro and Chone they are characterized by a production of less than 25 pounds per day in $70 \%$ of the farms only in Carmen the productions in $43 \%$ of the cases are between 25 and 50 pounds daily, $17 \%$ of the producers are willing to change salting method if this increases its performance.
\end{abstract}

Descriptors: Animal nutrition, cattle, dairy products, dairy industry.

\section{INTRODUCCIÓN}

El queso es un producto elaborado a partir de la leche de diversos mamíferos mayores domesticados por el ser humano, nutricionalmente se caracteriza por una alto contenido de proteína, que puede variar entre el $20-25 \%$ según su contenido graso y dureza(Galván, 2005).

Según la organización de las naciones unidas para la alimentación y la agricultura, durante el 2011 se produjeron cerca de 97500 toneladas métricas de queso a partir de 


\section{CIENCIAMATRIA}

Revista Interdisciplinaria de Humanidades, Educación, Ciencia y Tecnología

Año VI. Vol. VI. Nº1. Edición Especial. 2019

Hecho el depósito de ley: pp201602FA4721

ISSN-L: 2542-3029; ISSN: 2610-802X

Universidad Nacional Experimental Francisco de Miranda (UNEFM). Santa Ana de Coro. Venezuela

Rudyard Antonio Arteaga Solorzano; Freddy Alain Mendoza Rivadeneira; Roy Leonardo Barre Zambrano; Plinio Abelardo Vargas Zambrano

leche entera de vaca en el Ecuador, un $7.1 \%$ más que las 91000 toneladas reportadas en el 2010. (FAO, 2012)

El salado del queso fresco es una operación unitaria necesaria para mejoramiento de sabor, rendimiento e inocuidad del queso, se realiza después del desuerado, normalmente por adición directa en los quesos frescos (Padilla, 2002).

El salado puede ser realizado con diferentes ingredientes, tales como sal lodada comercial y la sal en grano que es usada principalmente por los productores más pequeños, esto debido a su disponibilidad. (MAGAP, 2012)

Los ingredientes usados por los productores medianos a grandes, suele ser sal industrial, las cual presenta ventajas en costos respecto a las antes mencionadas.

El presente estudio busca cuantificar por medio de encuestas y medición directa que procedimientos de salado se utiliza en la provincia de Manabí para la elaboración del queso fresco artesanal, así como dar una idea de la producción realizada en los dichos sectores productivos.

Este estudio brindará una perspectiva de los métodos de elaboración del queso artesanal en la zona de Manabí generando ideas generales de las opciones disponibles para le mejora continua de la matriz productiva.

\section{METODOLOGÍA}

La investigación fue de tipo descriptiva, siguiendo los postulados de Hernández, Fernández y Baptista (2014), se realizó en la zona norte de la provincia de ManabíEcuador, por ser la mayor productora de queso fresco artesanal de la costa ecuatoriana, dentro de la misma los cantones: Chone, El Carmen, Flavio Alfaro y Pedernales representan el $90 \%$ de esta producción de queso en la provincia. Para el presente estudio se consideraron los productores registrados en el proyecto de la CONEFA (Comisión Nacional de Erradicación de Fiebre Aftosa) 2016 de los cantones Chone, Flavio Alfaro, Pedernales y El Carmen) y, para lo cual se utilizó la siguiente ecuación de muestreo que cita Morales (2011), para población finita indicada para universo de entre 100-5000. 


$$
n=\frac{N}{1+\frac{e^{2}(N-1)}{Z^{2} p q}}
$$

Donde:

$\mathrm{n}$ = tamaño de muestra a estudiar

$\mathrm{N}$ = Población

$\mathrm{e}=$ Error muestral

$\mathrm{Z}=$ nivel de confianza

$\mathrm{p} \mathrm{q}=$ Varianza de la población

En el detalle del cuadro que se desarrolló a continuación resumen los datos y la muestra estimada que formó parte del estudio:

Cuadro 1

Determinación de muestra para observación in situ

\begin{tabular}{cccccc} 
Cantón & Población (N) & p q & Error (e) & $\mathbf{Z}$ & Muestra \\
\hline Chone & 236 & 0,5 & 0,5 & 1,96 & 147 \\
Flavio Alfaro & 283 & 0,5 & 0,5 & 1,96 & 163 \\
Pedernales & 187 & 0,5 & 0,5 & 1,96 & 126 \\
El Carmen & 185 & 0,5 & 0,5 & 1,96 & 126 \\
\hline
\end{tabular}




\section{VARIABLES EVALUADAS}

\section{Cuadro 4}

Descripción de las variables evaluadas.

\begin{tabular}{|c|c|c|c|}
\hline $\begin{array}{c}\text { Conceptualización } \\
\text { de las variables }\end{array}$ & $\begin{array}{c}\text { Dimensión o } \\
\text { Categoría }\end{array}$ & Indicador & Instrumentos \\
\hline & & $\begin{array}{ll}\text { - } & \text { En grano } \\
& \text { gruesa }\end{array}$ & \\
\hline TSU: & Variable & - En grano & Encuesta \\
\hline Tipo de Sal Usado & Dependiente & $\begin{array}{ll} & \text { fina } \\
\text { - } & \text { Refinada } \\
\text { - } & \text { Salmuera }\end{array}$ & Fotos \\
\hline $\begin{array}{c}\text { RUTS: } \\
\text { Razón de uso de } \\
\text { tipo de sal }\end{array}$ & $\begin{array}{c}\text { Variable } \\
\text { Dependiente }\end{array}$ & $\begin{array}{ll}\text { - } & \text { Económica } \\
\text { - } & \text { Rendimiento } \\
\text { - } & \text { Sanidad }\end{array}$ & Encuesta \\
\hline $\begin{array}{c}\text { MS: } \\
\text { Momento del } \\
\text { Salado }\end{array}$ & $\begin{array}{c}\text { Variable } \\
\text { Dependiente }\end{array}$ & $\begin{array}{l}\text { - Después del } \\
\text { desuerado } \\
\text { - En la } \\
\text { cuajada }\end{array}$ & $\begin{array}{c}\text { Encuesta } \\
\text { Fotos }\end{array}$ \\
\hline $\begin{array}{c}\text { CSU: } \\
\text { Cantidad de sal } \\
\text { utilizada }\end{array}$ & $\begin{array}{c}\text { Variable } \\
\text { Dependiente }\end{array}$ & $\begin{array}{ll}- & 0-10 \mathrm{gr} \\
- & 11-20 \mathrm{gr} \\
- & >20 \mathrm{gr}\end{array}$ & $\begin{array}{c}\text { Encuesta } \\
\text { Fotos }\end{array}$ \\
\hline $\begin{array}{c}\text { CQP: } \\
\text { Cantidad de queso } \\
\text { producido }\end{array}$ & $\begin{array}{c}\text { Variable } \\
\text { Dependiente }\end{array}$ & $\begin{array}{l}-\quad 1-25 \mathrm{lb} \\
-\quad 26-50 \mathrm{lb} \\
-\quad>50 \mathrm{lb}\end{array}$ & $\begin{array}{c}\text { Encuesta } \\
\text { Fotos }\end{array}$ \\
\hline DCT: & Variable & - Si & Encuesta \\
\hline Disposición a & Dependiente & - No & Fotos \\
\hline
\end{tabular}




\section{CIENCIAMATRIA}

Revista Interdisciplinaria de Humanidades, Educación, Ciencia y Tecnología

Año VI. Vol. VI. Nº1. Edición Especial. 2019

Hecho el depósito de ley: pp201602FA4721

ISSN-L: 2542-3029; ISSN: 2610-802X

Universidad Nacional Experimental Francisco de Miranda (UNEFM). Santa Ana de Coro. Venezuela

Rudyard Antonio Arteaga Solorzano; Freddy Alain Mendoza Rivadeneira; Roy Leonardo Barre Zambrano; Plinio Abelardo Vargas

cambiar de técnica

RPC:

Razón del posible cambio

Variable

Dependiente

LE:

Lugar de estudio
Zambrano

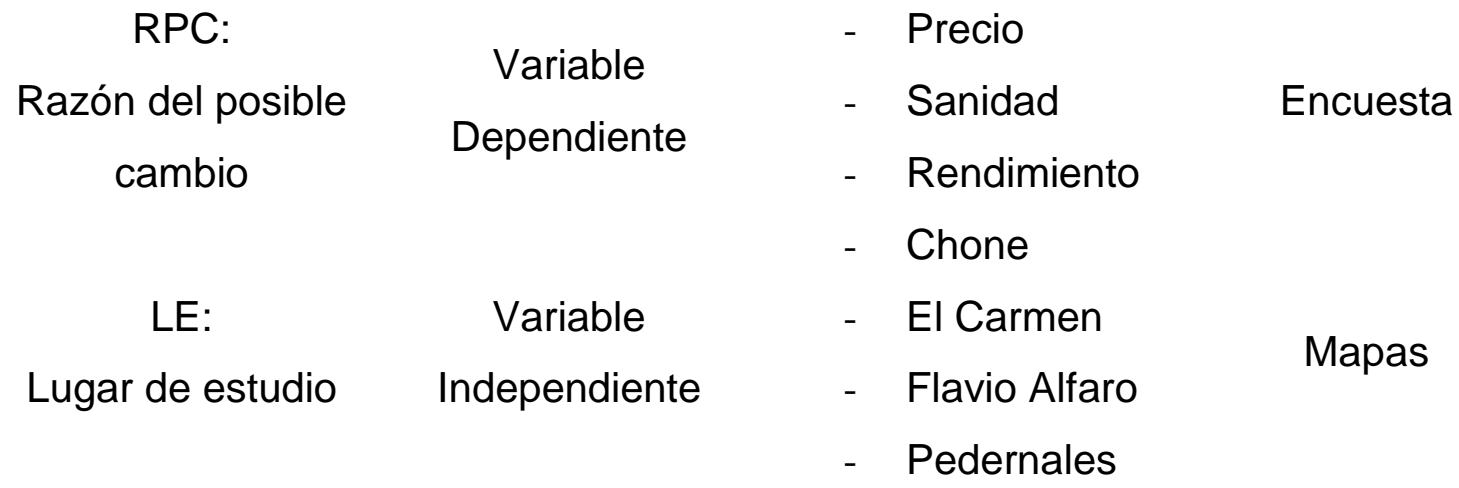

\section{RESULTADOS Y DISCUSIÓN}

\section{Gráfico 1}

Regiones según el tipo de cuajo usado expresado en fincas.

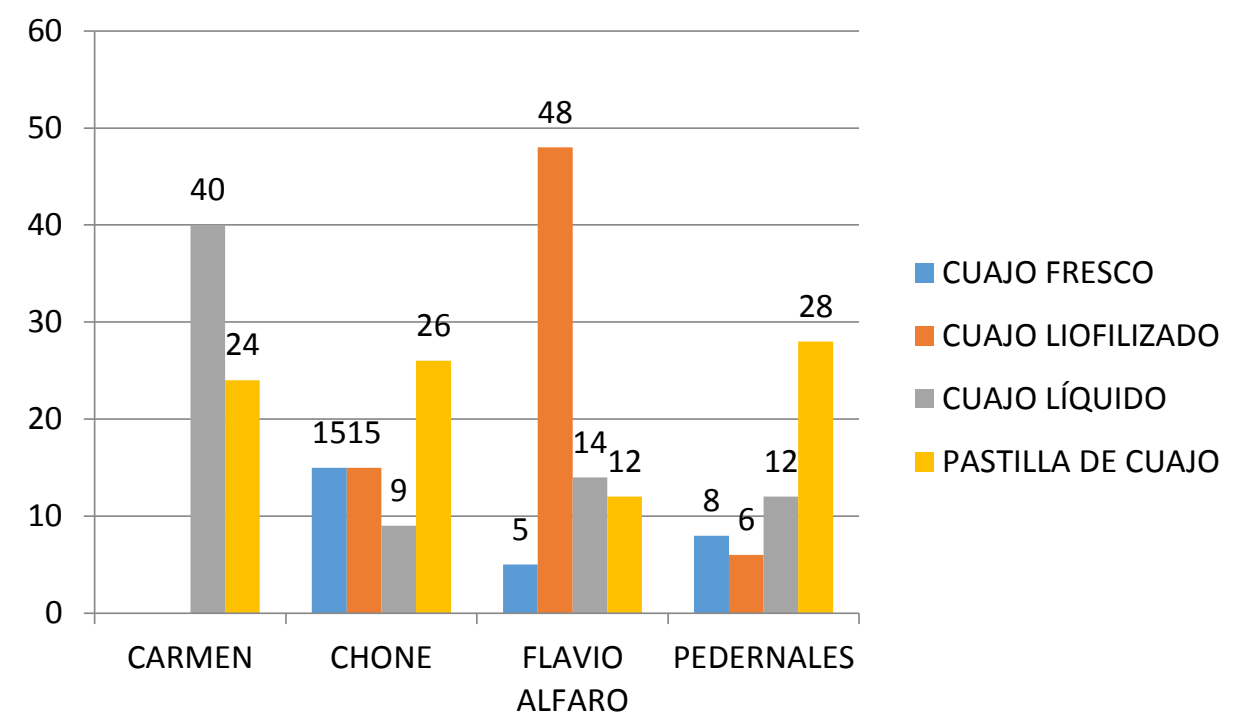

Como se puede observar en el gráfico 1 en El Carmen tiene 40 fincas que utilizan cuajo líquido, es decir el 62.5\% de la población prefiere usar cuajo líquido, siendo el único lugar de estudio donde la mayor parte de la población utiliza cuajo líquido. 


\section{CIENCIAMATRIA}

Revista Interdisciplinaria de Humanidades, Educación, Ciencia y Tecnología

Año VI. Vol. VI. N¹. Edición Especial. 2019

Hecho el depósito de ley: pp201602FA4721

ISSN-L: 2542-3029; ISSN: 2610-802X

Universidad Nacional Experimental Francisco de Miranda (UNEFM). Santa Ana de Coro. Venezuela

Rudyard Antonio Arteaga Solorzano; Freddy Alain Mendoza Rivadeneira; Roy Leonardo Barre Zambrano; Plinio Abelardo Vargas Zambrano

En Chone la preferencia más común es el cuajo en pastilla con el $40 \%$ de uso entre la población, superior al $23 \%$ de preferencia de cuajo fresco y cuajo liofilizado las cuales o el $14 \%$ de uso que tiene el cuajo líquido.

Flavio Alfaro presentó una preferencia del $61 \%$ con respecto al 18,15 y $6 \%$ que hubo para el cuajo líquido, la pastilla de cuajo y el cuajo fresco respectivamente; mientras que para Pedernales existió una preferencia mayor para la pastilla de cuajo donde un $52 \%$ de la población la eligió sobre las otras alternativas, que en ningún caso sobrepasan al 30 $\%$ de aceptación.

La preferencia de un tipo de coagulante sobre otros de forma tan marcada en cada uno de los lugares de estudio se explica con 2 factores: La disponibilidad geográfica de cada uno de los insumos y la asimilación de conceptos y adaptación a los mismos que se realiza como actividad de carácter social, haciendo que comunidades vecinas adapten métodos y costumbres de trabajo parecidas unas entre otras.

La preferencia en Manabí es de usar cuajo en pastilla, un 34\% de los productores lo usan, $29 \%$ de los productores usan cuajo líquido, $26 \%$ utilizan cuajo liofilizado y el $11 \%$ usan cuajo fresco.

\section{Gráfico 2}

Regiones según el momento de adición del cuajo

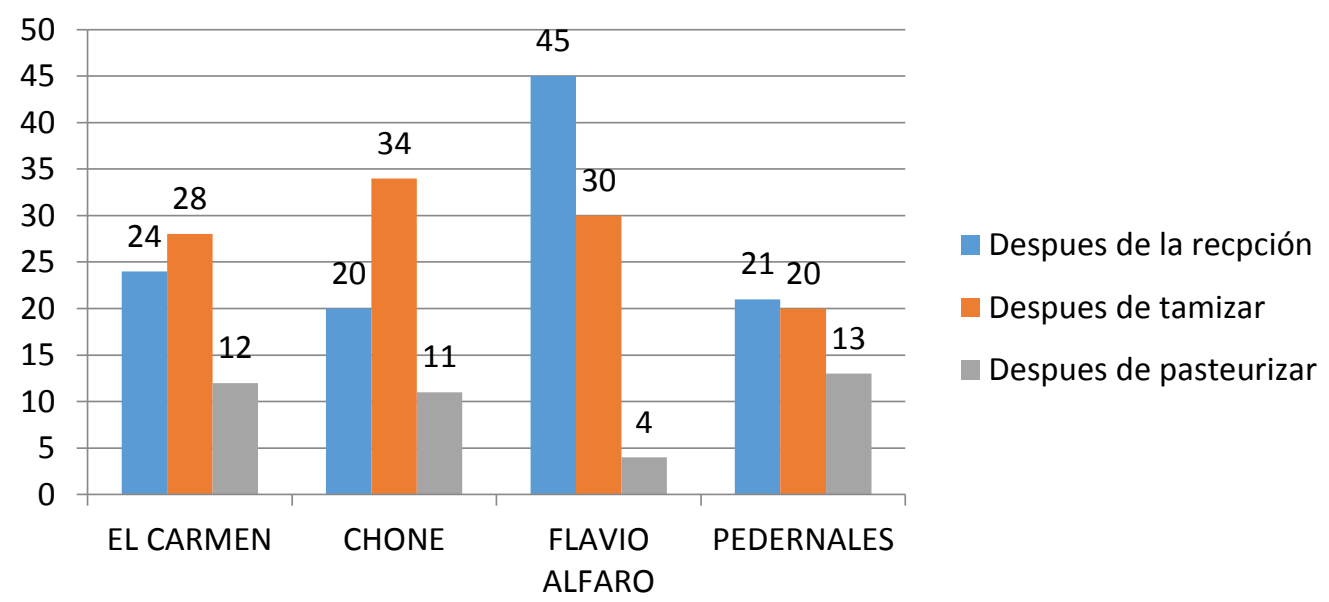




\section{CIENCIAMATRIA}

Revista Interdisciplinaria de Humanidades, Educación, Ciencia y Tecnología

Año VI. Vol. VI. Nº1. Edición Especial. 2019

Hecho el depósito de ley: pp201602FA4721

ISSN-L: 2542-3029; ISSN: 2610-802X

Universidad Nacional Experimental Francisco de Miranda (UNEFM). Santa Ana de Coro. Venezuela

Rudyard Antonio Arteaga Solorzano; Freddy Alain Mendoza Rivadeneira; Roy Leonardo Barre Zambrano; Plinio Abelardo Vargas Zambrano

Como en el gráfico 2 no existe preferencia por agregar el cuajo después del proceso de pasteurizado, que es el método más inocuo según Borjas (1998), esto se debe principalmente a que los productores carecen de la tecnología para pasteurizar la leche, el $100 \%$ de los productores que realizan el proceso de pasteurización agregan el cuajo después del mismo.

En Flavio Alfaro y Pedernales existe una preferencia para el método de agregar el cuajo después de la recepción del 56 y 38\%, mientras, que en el Carmen y Chone se agrega el cuajo después del filtrado en un 44 y $52 \%$ respectivamente.

Al no realizar un proceso de pasteurización de la leche el queso resultante tiene mayor carga bacteriana y un sabor característico, normalmente este proceso no se realiza en las producciones de queso en Manabí, solamente el 15\% de las fincas producen queso utilizando procesos de pasteurización.

\section{Gráfico 3}

Disposición a cambiar método de cuajado.

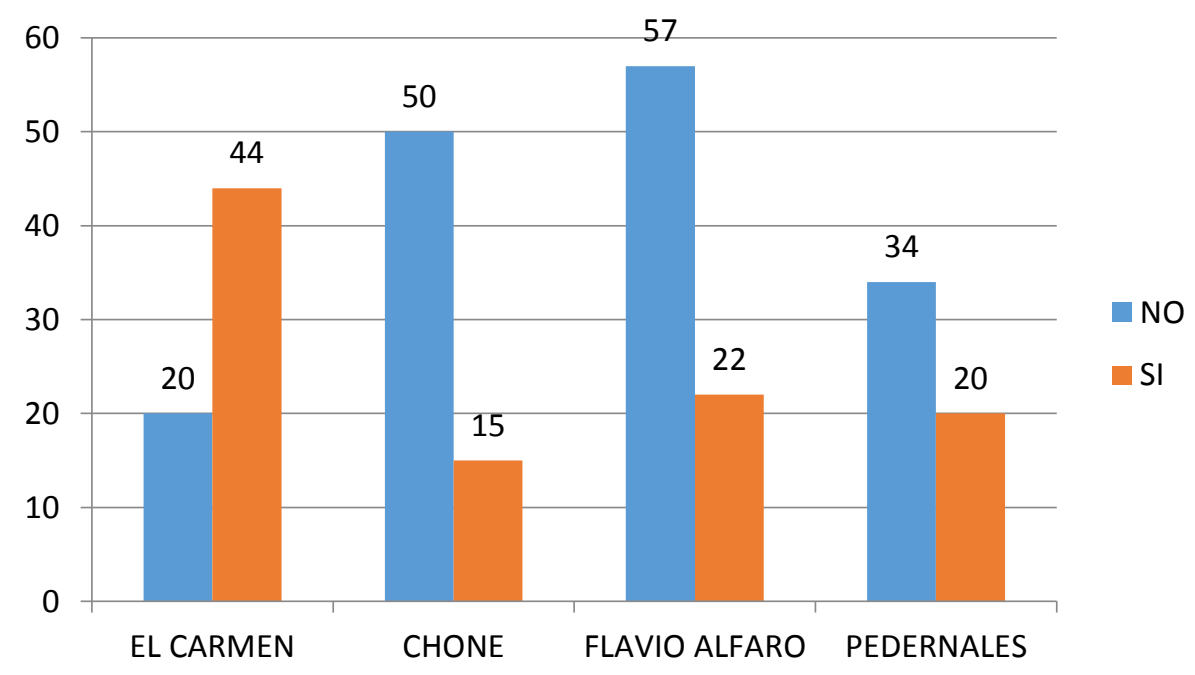




\section{CIENCIAMATRIA}

Revista Interdisciplinaria de Humanidades, Educación, Ciencia y Tecnología

Año VI. Vol. VI. Nº1. Edición Especial. 2019

Hecho el depósito de ley: pp201602FA4721

ISSN-L: 2542-3029; ISSN: 2610-802X

Universidad Nacional Experimental Francisco de Miranda (UNEFM). Santa Ana de Coro. Venezuela

Rudyard Antonio Arteaga Solorzano; Freddy Alain Mendoza Rivadeneira; Roy Leonardo Barre Zambrano; Plinio Abelardo Vargas Zambrano

Como se observa en el cuadro 3 solamente en El Carmen existe una mayoría de productores dispuestos a cambiar de método de cuajado en la elaboración de queso, es decir que el $69 \%$ de los productores están dispuestos a cambiar su método de cuajado, ya sea por hacer este más eficiente, aumentar la producción o porque están inconformes con el método que usan actualmente, desde una perspectiva global, el $61 \%$ de los productores no quieren cambiar de método usado debido a que actualmente están conformes con el que aplican.

\section{Gráfico 4}

Opinión de productores respecto a su método de cuajado

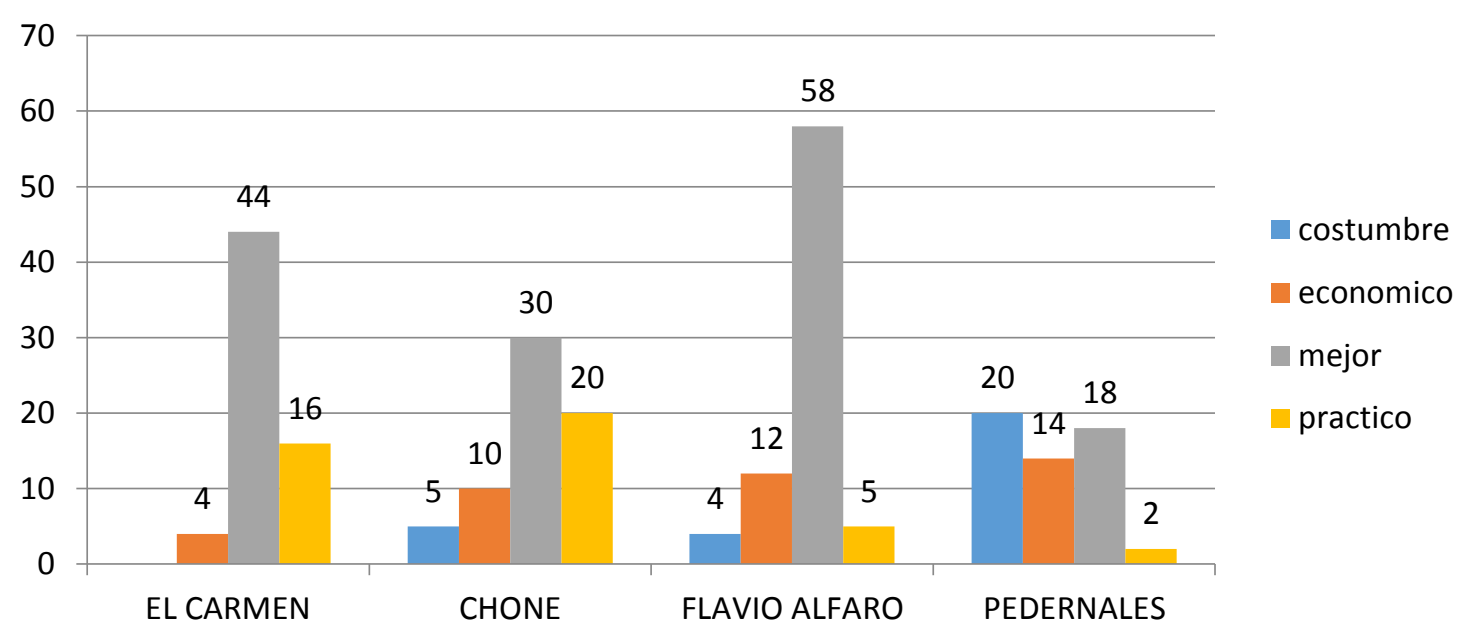

Como se observa en el gráfico 4 , la percepción del $57 \%$ de los productores es que el método que se encuentran usando actualmente es el mejor, lo cual concuerda con la información expresada en el gráfico 3 que expresa la renuencia general de los productores a cambiar método de elaboración de queso.

El método de cuajado en la producción de queso actualmente se encuentra en proceso de estancación debido a la falta de deseo de cambio entre los productores, con la única excepción del Carmen donde la mayoría de productores encuestados está dispuesto a 
cambiar de técnica, en un $68 \%$ de los casos para mejorar la producción en rendimiento y en $32 \%$ para mejorar la calidad e inocuidad de la misma.

La falta de tecnificación hace necesario la implementación de tecnologías que mejoren los procesos, se recomienda adoptar programas de centros de acopio lechero privados que generen producciones mejores como se plantea en el estudio de Guaman \& Lerdón (1999) donde se pudo integrar la producción de 60 de los cuales ninguno pasteurizaba la leche antes de procesarla en queso antes del proyecto.

\section{CONCLUSIONES}

En Manabí se usa principalmente el cuajo en pastilla en un 34\% de las producciones, el cual se agrega a leche no pasteurizada en un $85 \%$ de los casos.

Solo en El Carmen los productores se encuentran abiertos a cambiar su método de elaboración de queso, en el $69 \%$ de los casos desean métodos que mejorar el rendimiento de la producción.

El $15 \%$ de la leche que se produce en la Zona Norte de Manabí es pasteurizada.

\section{REFERENCIAS CONSULTADAS}

1. Aray, M., Intriago, M. \& Mendoza, H. (2013). Producción y comercialización de queso mozarella en la ciudad de Chone, provincia de Manabí. Tesis para obtar al título de Ingeniero Comercial, Departamento de Economía, Univerciada Técnica de Manabí, Manabí, Ecuador.

2. Borjas, E. (1998). Tecnificación de los procesos de manufactura y caracterización de quesos artesanales centroamericanos para exportación. Tesis para optar al título de Ingeniero Agroindustrial, Departamento de Agroindustria Alimentaria, Escuela Agrícola Panamericana el Zamorano, Francisco Morazan, Honduras.

3. Carmona, M. Rubio, C. \& Lemus, C. (2002). Estadística Aplicada a la Investigación. Nayarit: México

4. Correa, R., Glas, J., Muñoz, P., Vaca, C., Long, G., Arellano, H., Rivera, P., Poveda, R., Espinosa, R., Ramírez, R., Sánchez, M., Martínez, J., Chilán, C., Torre, P., Maji, R., Quiñónez, Y., Rodríguez, K. \& Larrea, A. (2013). Plan nacional 


\section{CIENCIAMATRIA}

Revista Interdisciplinaria de Humanidades, Educación, Ciencia y Tecnología

Año VI. Vol. VI. Nº1. Edición Especial. 2019

Hecho el depósito de ley: pp201602FA4721

ISSN-L: 2542-3029; ISSN: 2610-802X

Universidad Nacional Experimental Francisco de Miranda (UNEFM). Santa Ana de Coro. Venezuela

Rudyard Antonio Arteaga Solorzano; Freddy Alain Mendoza Rivadeneira; Roy Leonardo Barre Zambrano; Plinio Abelardo Vargas Zambrano

del buen vivir. Quíto: Gobierno de la República del Ecuador.

5. Bylund, G. (1995). Dairy processing handbook. Lund: Tetrapack.

6. FAO. (2012). Datos de producción de queso a partir de leche entera de vaca. Italia: FAO.

7. Galván, M. (2005). Proceso básico de la leche y el queso. Revista digital universitaria UNAM , 167-184.

8. García, O., Ochoa, I., Novoa, C., Baylon, C., Granados, F., Duque, O., \& Murcia, R. (1987). Derribados Lacteos: Procesamiento de quesos blancos. Bogota: Centro Agropecuario de la Sabana.

9. Hernández, R., Fernández, C., \& Baptista, P. (2014). Metodología de la investigación. México, Mc Graw Hill Hispanoamericana. Hill Internacional.

10. MAGAP. (2012). Sistema de Información Nacional de Agricultura, Ganadería Acuacultura y Pesca. Retrieved from Reporte de Resultados del Censo Provincial Completo en excel (Todas las Provincias): http://sinagap.agricultura.gob.ec/resultados-provinciales.

\section{REFERENCES CONSULTED}

1. Aray, M., Intriago, M. \& Mendoza, H. (2013). Production and marketing of mozzarella cheese in the city of Chone, province of Manabí. Thesis to obtain the title of Commercial Engineer, Department of Economics, Technical University of Manabí, Manabí, Ecuador.

2. Borjas, E. (1998). Technification of the manufacturing processes and characterization of Central American artisanal cheeses for export. Thesis to qualify for the degree of Agroindustrial Engineer, Department of Food Agribusiness, Pan American Agricultural School El Zamorano, Francisco Morazan, Honduras.

3. Carmona, M. Rubio, C. \& Lemus, C. (2002). Applied Statistics to Research. Nayarit: Mexico

4. Correa, R., Glas, J., Muñoz, P., Vaca, C., Long, G., Arellano, H., Rivera, P., Poveda, R., Espinosa, R., Ramírez, R ., Sánchez, M., Martínez, J., Chilán, C., 
Torre, P., Maji, R., Quiñónez, Y., Rodríguez, K. \& Larrea, A. (2013). National plan of good living. Chemical: Government of the Republic of Ecuador.

5. Bylund, G. (1995). Dairy processing handbook. Lund: Tetrapack.

6. FAO. (2012). Cheese production data from whole cow's milk. Italy: FAO.

7. Galván, M. (2005). Basic process of milk and cheese. University digital magazine UNAM, 167-184.

8. García, O., Ochoa, I., Novoa, C., Baylon, C., Granados, F., Duque, O., \& Murcia, R. (1987). Demolished Dairy Products: White cheese processing. Bogota: Agricultural Center of La Sabana.

9. Hernández, R., Fernández, C., \& Baptista, P. (2014). Investigation methodology. Mexico, Mc Graw Hill Hispanic American. Hill International

10. MAGAP. (2012). National Information System of Agriculture, Livestock Aquaculture and Fisheries. Retrieved from Report of Results of the Complete Provincial Census in excel (All Provinces): http://sinagap.agricultura.gob.ec/resultados-provinciales. 